\title{
Paired Mesoderm Homeobox Protein 2B
}

National Cancer Institute

\section{Source}

National Cancer Institute. Paired Mesoderm Homeobox Protein 2B. NCI Thesaurus.

Code $C 97726$.

Paired mesoderm homeobox protein 2B (314 aa, $32 \mathrm{kDa}$ ) is encoded by the human $\mathrm{PHOX} 2 \mathrm{~B}$ gene. This protein plays a role in the regulation of gene expression. 